\title{
Editorial
}

\section{LUCIO'S PHENOMENON: AN OVERVIEW}

This paper is intended for leprologists outside of North and Central America who have had little or, more likely, no clinical experience with Lucio's phenomenon. The purpose of this paper is to delineate the features which establish Lucio's phenomenon as a distinctive reactional state. The data base for this paper is the experience with Lucio's phenomenon at the University of Southern California/Los Angeles County Medical Center. This experience has been more elaborately detailed in three other publications: a retrospective chart study (Rea and Levan, 1978), a report of an immunologic investigation (Quismorio et al., 1978), and a report of a comparative histopathological study (Rea and Ridley, to be published). For further reading in English the paper of Latapi and Zamora (1948) and the monograph by Frenken (1963) are particularly recommended.

\section{Historical Background}

In 1852 Lucio and Alvarez described a necrotizing skin reaction complicating the course of non-nodular leprosy. Although this finding was confirmed by their contemporaries, knowledge of the reaction was lost until rediscovered by Latapi and his co-workers. Latapi and co-workers' contributions included recognition that histopathologically the necrotizing reaction was a vasculitis and that clinically the reaction was restricted to patients with a form of diffuse, non-nodular lepromatous leprosy. These investigators named the necrotizing reaction Lucio's phenomenon eponymically and erythema necroticans descriptively, and designated the form of diffuse lepromatous leprosy as pure and primitive diffuse lepromatous (PPDL) (Lapati and Zamora, 1948).

\section{Definition and Prevalence}

Any patient who had a clinically characteristic haemorrhagic infarct and diffuse, non-nodular lepromatous leprosy was considered as having Lucio's phenomenon. Eleven such individuals have been clinic patients in the past 9 years, approximately one out of 6 lepromatous patients.

\section{The Anlage, PPDL}

In this series patients with PPDL were truly without nodules. In 2 of our patients the earlobes were definitely thickened and in one, the only atypical 
case in the 11 patients, the skin of the forehead was folded, suggesting leonine faces, but without loss of normal skin markings. Total alopecia of eyebrows (and often of the eyelashes) was present in all. Acral distal symmetrical anaesthesia of some degree was also present in all. Trophic changes, attributable to sensory loss, were present in 3 patients, but motor palsies as evidenced by contractures of fingers, interosseous atrophy, or weakness were absent. Destructive rhinitis was present in all and septal perforation common. In contrast, only one patient demonstrated beading of a corneal nerve, the same atypical patient. A profound telangectasia of the face was present in one patient.

\section{The Reaction, Lucio's Phenomenon}

The earliest observed lesion was a slightly indurated light blue plaque with an erythematous halo. Lesions soon evolved into typical haemorrhagic inf arcts with or without bulla formation (Fig. 1). Lesions were painful but not tender, and arose in crops on the extremities. Large lesions below the knees frequently ulcerated. Small lesions below the knees and most other lesions elsewhere crusted, without overt ulcer formation but healed with scarring. The typical well-developed haemorrhagic infarct had a serrated border, with the convex margin lying within the lesion. Lesions ranged from $2 \mathrm{~mm}$ to $5 \mathrm{~cm}$ in diameter.

In this series, the initial onset of Lucio's phenomenon has not been observed in patients on dapsone. Three of the 11 patients had had dapsone prior to the onset of Lucio's phenomenon, but in all of these 3 dapsone had been stopped for at least 6 months before developing this reaction.

\section{SYSTEMIC SIGNS AND SYMPTOMS}

Fever was not associated with Lucio's phenomenon in these 11 patients, in marked contrast to patients with ENL (Rea and Levan, 1975). Arthritis was present in one patient, and nephritis in another, but it is uncertain if these changes were related to Lucio's phenomenon or even to lepromatous leprosy. Splenomegaly was present in 3 patients. Orchitis and iritis were not present.

\section{LABORATORY FINDINGS}

None of the 11 patients with Lucio's phenomenon had a leukocytosis or absolute neutrophilia, in contrast to patients with ENL (Rea and Levan, 1975).

Routine laboratory abnormalities which did not differ greatly from those of other lepromatous patients included a mild normochromic, normocytic anaemia, high erythrocyte sedimentation rate, hypergammaglobulinemia and positive cardio-lipin antigen tests for syphilis.

\section{HISTOLOGICAL CHANGES}

Early lesions of Lucio's phenomenon showed ischaemic epidermal necrosis, necrosis in the walls of superficial vessels of the dermis, and endothelial proliferation in the medium-sized vessels of the mid-dermis (Figs 2 and 3).

Abundant AFB were found in endothelial cells, both in proliferating and in apparently normal cells (Fig. 4). These large numbers of AFB in endothelial 
cells have been emphasized by others (Obermayer et al., 1949; Derbes et al., 1960; Donner and Shively, 1967; Kramarsky et al., 1968).

Granuloma development in the dermis was variable, particularly in the superficial dermis; in two cases no perivascular granulomas were found.

Using the system of Ridley (1974) patients with Lucio's phenomenon were classified as lepromatous (LL). Because of the inflammatory infiltrate associated with the Lucio reaction, it was not possible to distinguish between polar (LLp) and subpolar (LLs) disease.

\section{IMMUNOLOGIC RESPONSES}

No immediate responsivity to lepromin was found in the four patients tested, the only finding at variance with the report of Latapi and Zamora (1948). (The variable composition of lepromin is a likely explanation for this difference.) No Mitsuta type of lepromin responsivity was found.

Tuberculin responsivity did not differ from that of controls, but dinitrofluorobenzene and dinitrochlorobenzene responsivity was slightly (statistically insignificantly) diminished.

Direct immunofluorescence studies of skin lesions demonstrated immunoglobulin and complement components in vessel walls (Quismorio et al., 1978), changes not demonstrable in patients with ENL (Rea et al., 1977).

Evidence for circulating immune complexes in the serum of patients with Lucio's phenomenon included positive Raji cell tests, positive latex fixation tests and cryoglobulinaemia, up to $37 \mathrm{mg} / 100 \mathrm{ml}$. The cryoglobulins were of the mixed type, consisting of $\operatorname{IgG}, \operatorname{IgM}, \operatorname{IgA}$ and complement components.

\section{THERAPEUTIC RESPONSES}

Of the 10 patients observed for 3 months or more, in 7 new lesions of Lucio's phenomenon ceased in association with dapsone as the sole therapeutic agent. Three patients continued to have new lesions while on dapsone; none of these 3 remitted in association with thalidomide therapy, but in each case new lesions ceased within one week of beginning rifampin.

Ulcerated lesions began to heal within 6 weeks of beginning antilepromatous chemotherapy and were completely re-epithelized by 6 months; only bland topical therapy was used.

\section{Perspectives}

Lucio's phenomenon is a distinctive reactional state as judged by clinical; histopathological and therapeutic criteria. The reaction occurs in patients with a variant of lepromatous leprosy, PPDL. Circulating immune complexes are associated with Lucio's phenomenon and may be important in its pathogenesis.

The restriction of Lucio's phenomenon to patients with PPDL can be partially understood by hypothesizing that these patients have a singularly deficient defence mechanism (Rea and Ridley, to be published). Thus this nadir of resistance permits the replication of $M$. leprae in endothelial cells, enhancing the exposure of bacterial antigen to circulating antibody, eventually resulting in 


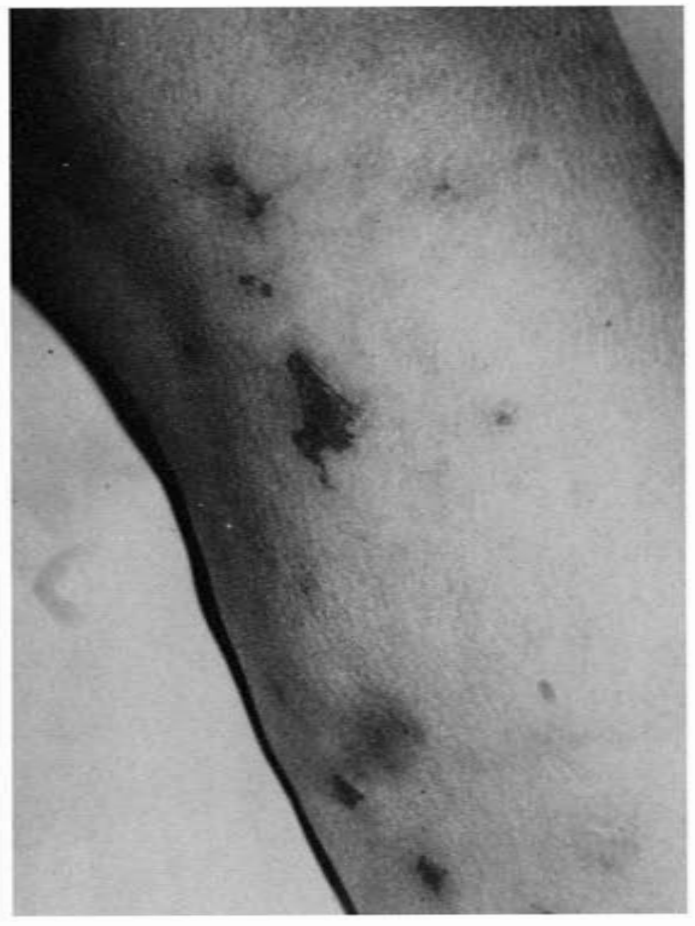

Fig. 1. Well developed haemorrhagic lesions of the Lucio reaction. Note the variation in size and the serrated margins.

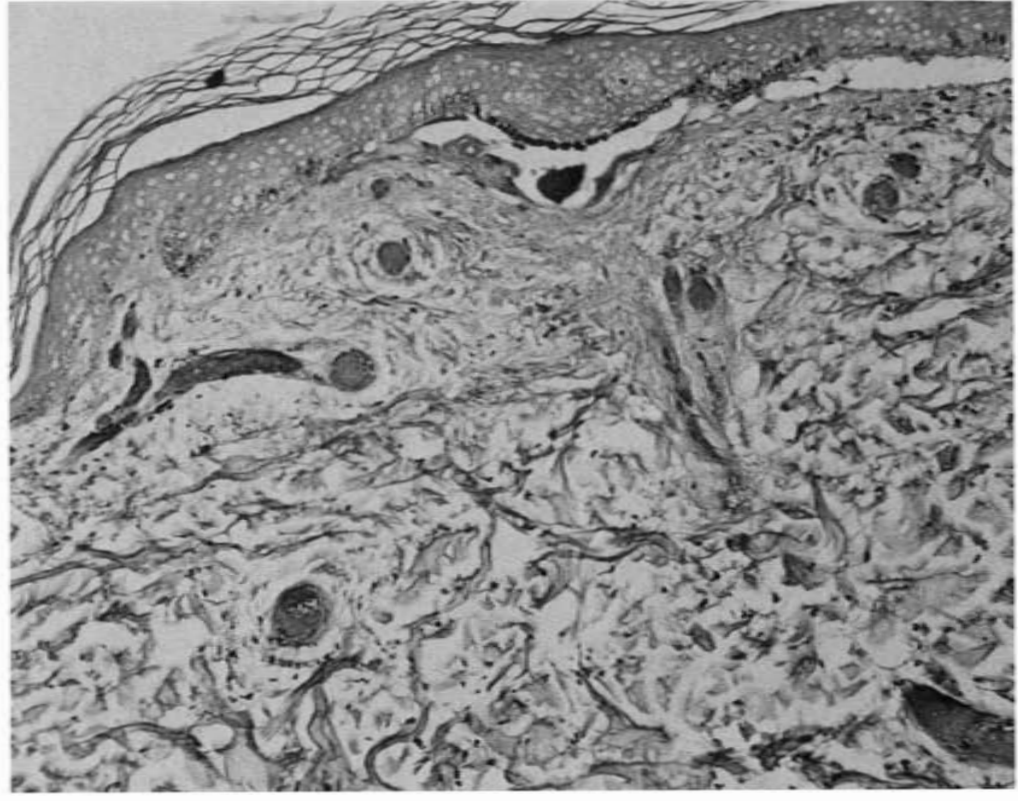

Fig. 2. Ischaemic epidermal necrosis and necrosis of vessel walls is demonstrated by loss of nuclear detail. Note the intense passive congestion of the necrotic vessels. Haematoxylin and eosin. $\times 150$. 


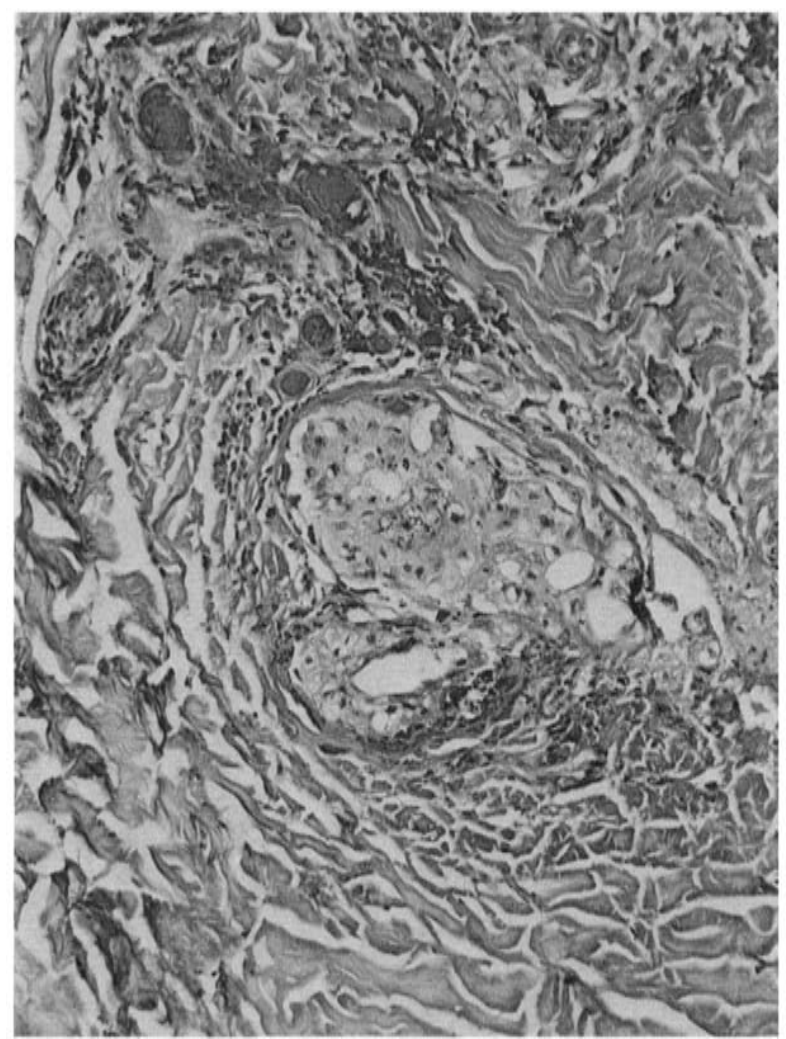

Fig. 3. Well developed endothelial proliferation in a mid-dermal vessel. Note the sparse inflammatory infiltrate and the absence of granuloma development. Haematoxylin and eosin. $\times 150$.

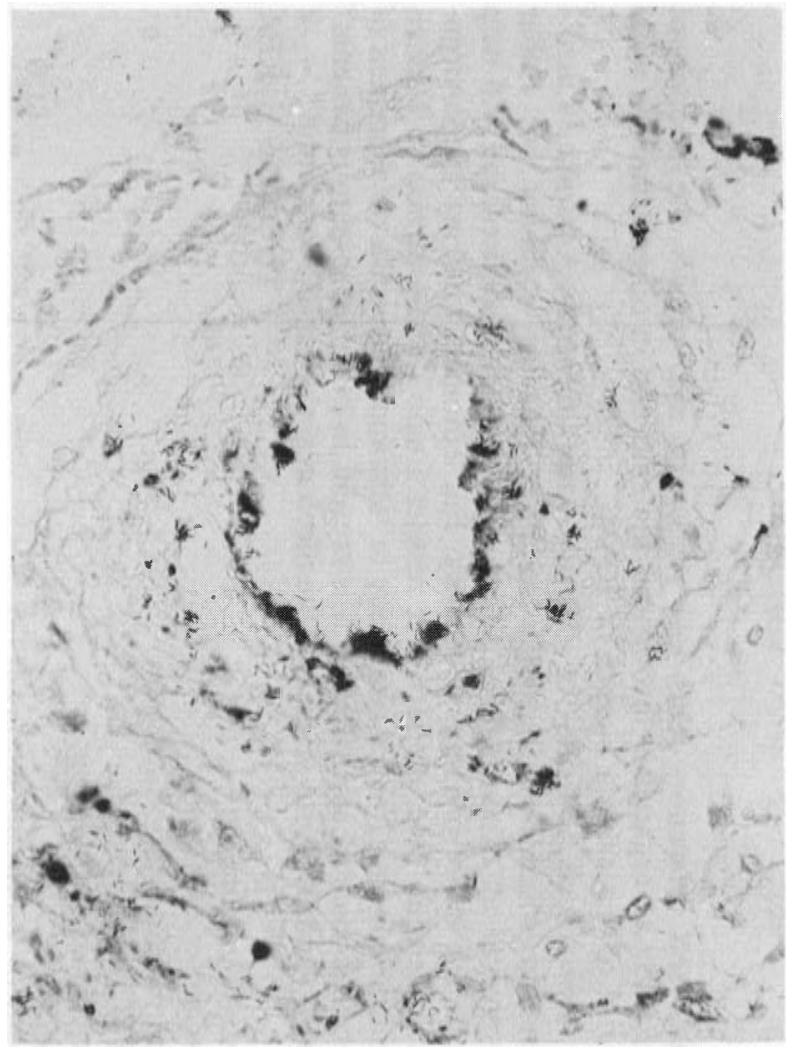

Fig. 4. Abundant AFB in endothelial cells of a vessel which appeared to be normal on routine section. Fite-Faraco. $\times 300$. 
vasculitis and infarction. This low resistance also allows the unopposed dissemination of organisms throughout the skin and in other tissues as well, giving rise to the clinical picture of PPDL.

For leprologists outside of Mexico and Central America, Lucio's phenomenon should not be regarded as a faraway curiosity but rather approached as an experiment of nature, one that when understood will help illuminate the entire problem of leprosy. For example, what are the environmental or genetic determinants that restrict Lucio's phenomenon to Mexico and Central America? What deficiency in resistance allows the development of Lucio's phenomenon and PPDL but is present in other lepromatous patients? Why do no nodules form or what is the mechanism of nodule formation?

THOMAS H. REA

Section of Dermatology

Department of Medicine

University of Southern California

School of Medicine, and

Department of Dermatology

Los Angeles County

University of Southern California

Medical Center

Los Angeles, California, USA

\section{References}

Derbes, V. J., Samuels, M., Williams, O. P. and Walsh, J. J. (1960). Diffuse leprosy: case in a Louisiana negro. Arch. Derm. 81, 210.

Donner, R. S. and Shively, J. A. (1967). The "Lucio phenomenon" in diffuse leprosy. Ann. intern. Med. 67, 831 .

Frenken, J. H. (1963). Diffuse Leprosy of Lucio and Latapi. Translated by H. de KeijzerJacobowitz, J. M. H. Frenken and E. D. Fowler. Orangestad, Arube Netherlands Antilles, De Wit, Inc.

Kramarsky, B., Edmondson, H. A., Peters, R. L. and Reynolds, T. B. (1968). Lepromatous leprosy in reaction. Arch. Path. 85, 516.

Latapi, F. and Zamora, A. C. (1948). The "spotted" leprosy of Lucio (La lepra "manchada" de Lucio). Int. J. Lepr. 16, 421.

Obermayer, M. E., Bonar, S. C. and Rosenquist, R. (1949). Diffuse lepra. J. invest. Derm. 12, 243.

Quismorio, F. P., Rea, T., Chandor, S., Levan, N. and Friou, G. (1978). Lucio's phenomenon: an immune complex deposition syndrome in lepromatous leprosy. J. Clin. Immun. Immunopath. 9, 184.

Rea, T. H. and Levan, N. E. (1975). Erythema nodosum leprosum in a large general hospital. Arch. Derm. 111, 1575.

Rea, T. H. and Levan, N. E. (1978). Lucio's phenomenon and diff use nonnodular lepromatous leprosy. Arch. Derm. 114, 1023

Rea, T. H., Quismorio, F. P., Levan, N. E. and Friou, G. J. (1977). Letter: Erythema nodosum leprosum. Arch. Derm. 113, 234.

Rea, T. H. and Ridley, D. S. Lucio's phenomenon: a comparative histological study. Int. J. Lepr. (To be published).

Ridley, D. S. (1974). Histological classification and the immunological spectrum of leprosy. Bull. WHO 51, 451. 